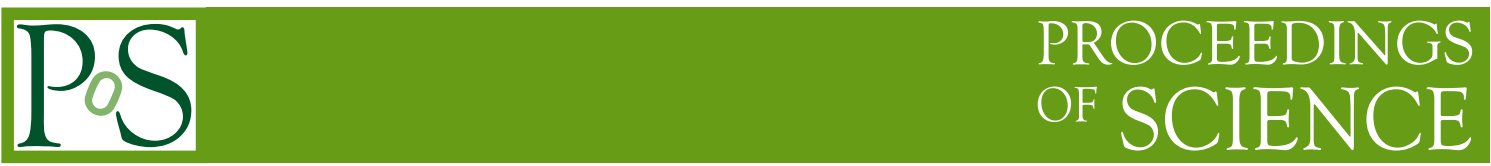

\title{
EDELWEISS-2 Dark Matter Search: recent results with new detectors
}

\author{
V. Yu. Kozlov* (for the Edelweiss collaboration) \\ Forschungszentrum Karlsruhe, Institut für Kernphysik, Postfach 3640, 76021 Karlsruhe, Germany \\ E-mail: Valentin.Kozlov@ik.fzk.de
}

EDELWEISS-2 is a direct dark matter (DM) search experiment using cryogenic Germanium bolometers. The most promising DM candidate, a so-called weakly interacting massive particle, WIMP, is expected to scatter off the target nuclei thus depositing a tiny energy in the detectors. A powerful event selection and good background knowledge are of crucial importance in this case. The experiment is situated in the French-Italian Fréjus tunnel, in the Modane underground laboratory LSM with a shielding of 4800 m.w.e. against cosmic rays. EDELWEISS is taking data since the end of 2007. The status of the experiment and the latest results are presented. Special emphasis is given to the performance of recently developed detectors. These detectors show a significantly improved beta/gamma rejection power and provide a promising base for next generation direct DM searches. In addition, the identification of muon-induced background events and special measurements of muon-induced neutrons are discussed.

European Physical Society Europhysics Conference on High Energy Physics, EPS-HEP 2009,

July 16 - 222009

Krakow, Poland

\footnotetext{
* Speaker.
} 


\section{Introduction}

A large number of cosmological observations require the existence of non-visible matter in the Universe of as yet unknown nature. An excellent particle candidate for this dark matter (DM) arises from the models of supersymmetry (SUSY) and it has a general name of weakly interacting massive particle (WIMP). Direct searches of WIMP's are based on measuring the energy of a recoiling nucleus originating from the WIMP-nucleus elastic scattering. This recoil energy calculated within the typically accepted models is in the range of 1 to $100 \mathrm{keV}$ and the expected interaction rate is below 0.01 event per day and per kilogram of the target material. In order to be sensitive to this low count rate at such energies, the experiments on direct DM search are commonly located deep underground to reduce influence of the cosmic rays, select low-radioactivity materials to be used, develop methods and the detectors themselves to have a very powerful background rejection, study general background conditions of the experiment.

\section{Edelweiss experiment}

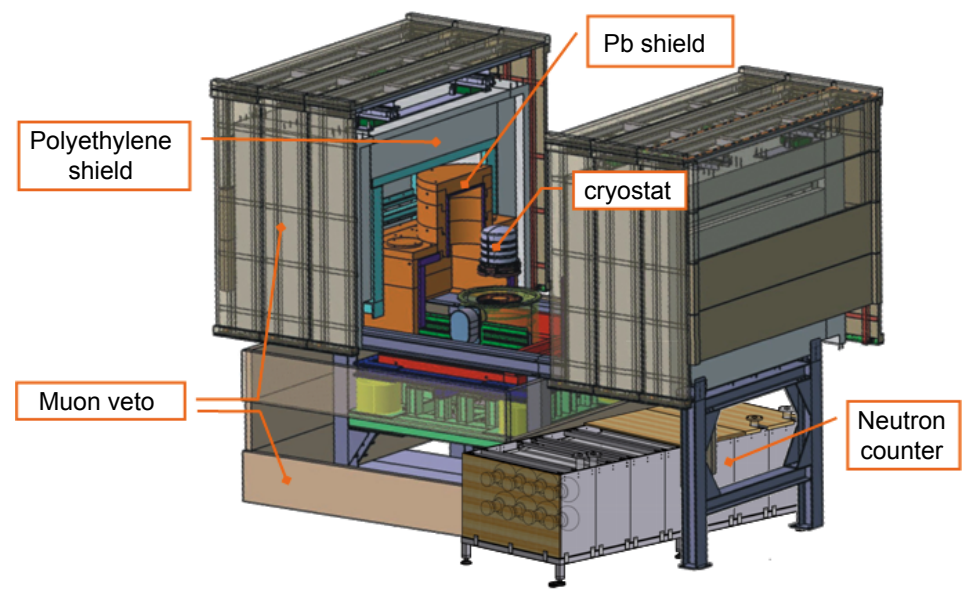

Figure 1: General scheme of the Edelweiss-2 experimental set-up.

Edelweiss-2 set-up (Fig. 1) is situated in the Laboratoire Souterrain de Modane (LSM), an underground lab in the Fréjus French-Italian road tunnel in the Alps. It profits from a shielding of 4800 m.w.e., which reduces the $\mu$-flux down to about $4 \mu / \mathrm{m}^{2} /$ day. The central part of the experiment are bolometers of pure natural Germanium, which serve as a target medium. Once these detectors are cooled down to about $20 \mathrm{mK}$, a deposited energy of a particle leads to two measurable processes: ionization and a temperature rise. The ratio of these signals, so-called Q-value, is different for nuclear and electron recoils, being respectively 0.3 and 1 , thus allowing a powerful background rejection. A general overview of the set-up is shown in Fig. 1. The dilution cryostat of the experiment can hold up to 110 detectors. It is surrounded by $20 \mathrm{~cm}$ of lead shield against an external $\gamma / \beta$-background and then by $50 \mathrm{~cm}$ of polyethylene to moderate neutrons. A muon veto system ( $>98 \%$ coverage) to tag remaining cosmic muons completes the installation. In addition, this muon veto system in combination with a recently installed neutron counter [5] allows to specifically study $\mu$-induced neutron background. The scientific goal of Edelweiss- 2 is to reach a sensitivity of better than $10^{-8} \mathrm{pb}$ for the spin-independent (SI) cross-section by 2012. 


\section{Bolometer improvements and the results}

Edelweiss-2 started to operate at the end of 2007, using first the standard Edelweiss-1 [1] bolometers. Although the background conditions were improved in comparison to Edelweiss- 1 by a factor of 3 for $\gamma$ 's and a factor of 2 in contamination of ${ }^{210} \mathrm{~Pb}$, three events were found in the region of interest (ROI) after an exposure of $93.5 \mathrm{~kg}$ day. These events, however, are attributed to so-called surface events [2], which appear in ROI due to an incomplete charge collection near the detector surface. Inability to identify such events makes these bolometers unsuitable for the goal of the experiment and thus a new type of Ge bolometers [2] was developed within the collaboration. A main principle of these detectors, named inter-digitized electrode detectors, or ID's, is to have concentric ring electrodes for charge collection instead of one plain electrode. By tuning the electric potentials applied on the neighboring electrodes one can reliably reject the near-surface events [2]. A first bolometer of this kind was installed in November 2007 having $200 \mathrm{~g}$ mass with 50\% as fiducial volume. The dedicated tests performed with ${ }^{133} \mathrm{Ba}$ calibration source showed that from $10^{5} \gamma$ 's no signal is obtained in ROI, which is equivalent to the exposure of $10^{3} \mathrm{~kg}$ day. Similar, when ${ }^{210} \mathrm{~Pb}$ source was fitted to one of the detectors ( $200 \mathrm{~g}$ ), from $1.210^{6} \beta$ 's recorded above $15 \mathrm{keV}$ threshold only 3 survived the selection cuts, which corresponds to $310^{4} \mathrm{~kg}$ day [2]. This satisfies well the needed background suppression for the aimed sensitivity. Furthermore, the first data taken with 2 ID detectors for the WIMP search show no evidence of events leaking from the electron-recoil band to either the nuclear recoil band or around (Fig.2 (left)). As for March 2009, operating two ID detectors (400 g \& $320 \mathrm{~g}$ ) above the
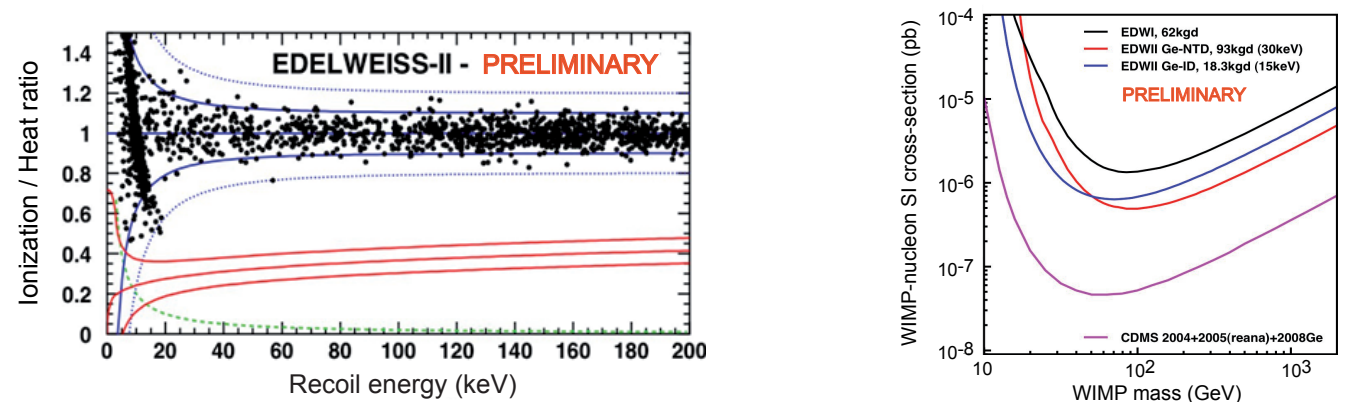

Figure 2: Recent results of the Edelweiss-2 experiment: data obtained in the physics run with a new ID bolometer (left) and the exclusion plots for the WIMP search, $93.5 \mathrm{~kg}$ day exposure with the old type detectors and $18.3 \mathrm{~kg}$ day exposure with 2 new ID's (right).

threshold of $15 \mathrm{keV}$ and with the exposure of $18.3 \mathrm{~kg}$ day allowed us to set a limit of $7 \cdot 10^{-7} \mathrm{pb}$ for the SI cross-section (Fig.2 (right)). Ten ID detectors are currently taking data since January 2009 and a factor of 20 higher sensitivity is expected by early 2010. Further production and R\&D for these bolometers continues.

\section{Studies of the muon-induced background}

Cosmic muons and especially muon-induced neutrons constitute an important background component for direct DM search. In order to understand this background in detail, comprehensive GEANT4 simulations were developed [3]. These simulations, for instance, show that while a muon interaction leads to a production of both electromagnetic and hadronic showers, the induced bolometer events with a deposited energy of $\mathrm{E} \geq 50 \mathrm{keV}$ are dominantly the electron recoils while events with 
$\mathrm{E} \leq 50 \mathrm{keV}$ are neutron recoils (Fig. 3 (left)). Analysis of real data measured in coincidence between the muon veto and bolometers [4] highlights this fact experimentally (Fig. 3 (right)). A rather limited statistics though makes it difficult to study this background by using the bolometers and the muon veto only. Thus a special neutron counter based on a liquid Gd-loaded scintillator was designed and installed in fall 2008 [5].
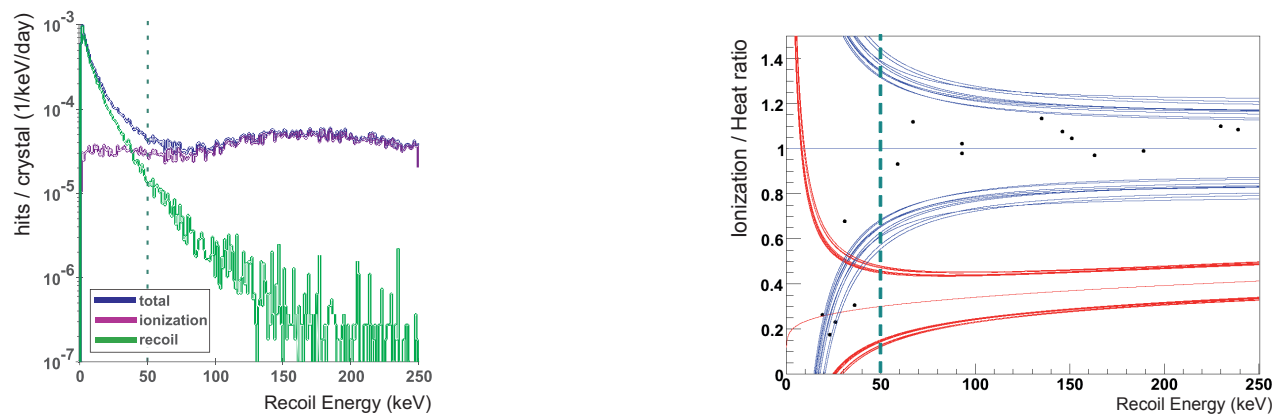

Figure 3: Energy deposited in the bolometers in case of the muon-induced events, GEANT4 simulations (left) and the experimentally measured events with a requirement of the muon veto - bolometer coincidence, half year data taking, $283 \mathrm{~kg}$ day exposure (right).

\section{Conclusion and acknowledgments}

Edelweiss-2 experiment successfully operates since end of 2007. New bolometers for direct DM search have been developed. These detectors have significantly improved efficiency of surface event rejection and are robustly fabricated. This development together with the detailed studies of the background conditions in LSM, in particular, muon induced neutrons give us the confidence to reach aimed sensitivity of better than $10^{-8} \mathrm{pb}$ for SI cross-section and put a good base for a DM experiment of next generation, e.g. EURECA [6], a 1-ton cryogenic detector array.

The detector development project is supported in part by Agence Nationale pour la Recherche under contract ANR-06-BLAN-0376-01. The studies of the muon-induced neutron background are in part supported by the German Research Foundation (DFG) through the Transregional Collaborative Research Center SFB-TR27 as well as by the EU contract RII3-CT-2004-506222.

\section{References}

[1] V. Sanglard et al., Phys. Rev. D71, 122002 (2005).

[2] A. Broniatowski et al., arXiv:0905.0753v1 [astro-ph.IM] and references there in; submitted to Phys. Lett. B.

[3] M. Horn, Simulations of the muon-induced neutron background of the EDELWEISS-II experiment for Dark Matter search, Ph.D. thesis, Universität Karlsruhe (TH), 2007 and FZKA scientific report 7391 (http://bibliothek.fzk.de/zb/berichte/FZKA7391.pdf)

[4] A. Chantelauze for the Edelweiss Collaboration, SUSY2007 Karlsruhe, Germany, 2007; arXiv:0710.5849v1 [astro-ph]; Ph.D. thesis in preparation.

[5] V.Yu. Kozlov for the Edelweiss Collaboration, IDM08 Stockholm, Sweden, 2008, PoS (idm2008) 086 (http://pos.sissa.it); arXiv:0902.4858v1 [astro-ph.IM].

[6] H. Kraus et al., Nucl. Phys. B (Proc. Suppl.) 173, 168 (2007). 